\title{
Commentary \\ Clinical trial update: implications and management of residual disease after neoadjuvant therapy for breast cancer
}

\author{
Erica L Mayer ${ }^{1}$, Lisa A Carey ${ }^{2}$ and Harold J Burstein ${ }^{1}$
}

${ }^{1}$ Dana-Farber Cancer Institute, 44 Binney Street, Boston, MA 02115, USA

${ }^{2}$ Lineberger Cancer Center, University of North Carolina, Chapel Hill, Chapel Hill, NC 27599, USA

Corresponding author: Erica L Mayer, emayer@partners.org

Published: 12 September 2007

This article is online at http://breast-cancer-research.com/content/9/5/110

(c) 2007 BioMed Central Ltd
Breast Cancer Research 2007, 9:110 (doi:10.1186/bcr1755)

adjuvant demonstrated no differences in either disease-free survival (DFS) or overall survival (OS). However, when segregated by pathologic response to treatment with neoadjuvant, individuals achieving a pCR experienced significantly improved outcomes compared with non-pCR subjects, including 9-year DFS (75\% versus 58\%) and OS (85\% versus $73 \%$ ), and a $50 \%$ decrease in the risk of death compared with all other pathologic outcomes (relative risk $0.50,95 \%$ confidence interval 0.32 to 0.78 ) [2,3]. In a similar study, the European Organization for Research and Treatment of Cancer (EORTC) randomized 698 subjects to anthracycline-based chemotherapy before or after surgery. Again, despite there being no differences in DFS or OS between the neoadjuvant and adjuvant groups, the pCR subgroup, defined as no residual invasive tumor in breast or lymph node tissue, did show improved OS (hazard ratio (HR) $0.86,95 \%$ confidence interval 0.77 to $0.96, P=0.008$ ) [4]. To investigate the role of exposure to neoadjuvant taxane, the NSABP randomized 2,411 subjects in study B-27 to neoadjuvant $A C$ alone, to neoadjuvant $A C$ and docetaxel before surgery, or to neoadjuvant $A C$ with adjuvant docetaxel after surgery. The addition of the preoperative taxane led to an increased rate of pCR (26\% with docetaxel versus $14 \%$ without docetaxel, $P<0.001)$; because pCR was defined in the breast tissue only, removing the $15 \%$ of patients categorized as pCR with continued lymph node involvement leads to true $\mathrm{pCR}$ rates of $21.8 \%$ with docetaxel versus $11.5 \%$ without docetaxel [5]. At a median follow-up of 6.5 years, individuals achieving $\mathrm{pCR}$ demonstrated significantly improved survival compared with those without $\mathrm{pCR}$ (DFS HR 0.45, $P<0.001$; OS HR 0.33, $P<0.0001$ ) [6].

Efforts have been made to identify more accurately patients who are more likely to achieve pCR with neoadjuvant chemo-

$\mathrm{AC}=$ adriamycin and cyclophosphamide; $\mathrm{DFS}=$ disease-free survival; $\mathrm{HR}=$ hazard ratio; $\mathrm{NX}=$ vinorelbine and capecitabine; OS = overall survival; $\mathrm{pCR}=$ pathologic complete response; RCB = residual cancer burden; TAC = docetaxel, doxorubicin, cyclophosphamide; VACP = vincristine, doxorubicin, cyclophosphamide, and prednisone; $\mathrm{VbMP}=$ vinblastine, methotrexate, and prednisone. 
therapy. Historical experience suggests that hormonereceptor negative tumors are far more likely to achieve pCR than hormone-receptor positive tumors (20\% versus $5 \%$ in a retrospective analysis) [7], and similarly ductal carcinomas may often achieve $\mathrm{pCR}$ whereas lobular carcinomas rarely do (less than $3 \%$ in larger experiences with up to 100 patients). A recently reported nomogram uses clinical and pathologic variables to predict likelihood of $\mathrm{pCR}$ in the breast and axilla with neoadjuvant anthracycline-based treatment. This nomogram includes independent predictors such as clinical stage at diagnosis, hormone receptor status, histologic grade, and number of preoperative chemotherapy cycles, and is able to predict $\mathrm{pCR}$ with reasonable accuracy [8].

Molecular assays have also been used to predict the likelihood of $\mathrm{pCR}$ in patients receiving neoadjuvant chemotherapy. The 21 -gene recurrence score assay, which quantifies the levels of expression of 16 genes involved in breast cancer pathogenesis, has been shown to predict whether patients are likely to achieve pCR in response to anthracycline- and taxane-based chemotherapy [9]. Tumors with low recurrence scores, which tend to have higher levels of hormone receptor expression, lower levels of HER2 expression, and lower grade, are far less likely to reach pCR than tumors with higher recurrence scores. Gene expression arrays can also classify tumor specimens into several subgroups, including the hormone-receptor positive luminal subtypes $A$ and $B$, as well as the HER2 ${ }^{+} /$ER $^{-}$(where ER is estrogen receptor) subtype and the basal-like subtype, which typically underexpresses both hormone receptors and Her2; variable prognoses are observed for different subtypes [10-12]. Microarray analysis of preoperative samples before neoadjuvant anthracycline-based chemotherapy has demonstrated significant differences in the rates of pCR among the four molecular subtypes, with the highest rates of $\mathrm{pCR}$ seen in the basal-like and HER2 ${ }^{+}$subgroups (45\%) and lower rates for the luminal tumors (6\%) [13]. Among the hormonereceptor negative patients who fail to achieve $\mathrm{PCR}$, there is a particularly high rate of early recurrence [14].

Although a lack of pCR portends a worse outcome, developing evidence suggests that there may be heterogeneity in outcome within the non-pCR group. Residual cancer burden (RCB), an index calculated from measurements of residual disease size and cellularity in the primary tumor and nodal metastases, has been evaluated in patients participating in two studies of neoadjuvant chemotherapy at the MD Anderson Cancer Center. Using a three-tier grading system, RCB was found to be a continuous predictor of DFS, with the patients at highest risk having the highest category of RCB [15]. Furthermore, expression array analysis of samples from NSABP B-27 demonstrates segregation of the non-pCR group into low-risk and high-risk categories, with differential survival outcomes [16]. Future use of these novel prognostic tools may better discriminate which patients with residual disease should receive further treatment from those with a favorable enough prognosis to defer further therapy.

Currently, for most patients who do not achieve pCR after treatment with a well-studied neoadjuvant chemotherapy regimen [17], there is no evidence to support the use of further adjuvant chemotherapy. The only exception would be a patient who received only a portion of the chemotherapy regimen (for example a patient who received four cycles of $A C$ and could go on to receive four additional cycles of a taxane). For the patient who has received a complete course of chemotherapy, any additional chemotherapy should be offered in the context of a clinical trial. Patients with hormone-receptor positive or Her2-positive disease certainly should receive appropriate adjuvant endocrine or trastuzumab therapy. Considering that residual disease may reflect resistance to the neoadjuvant chemotherapy, pilot trials have attempted to offer further non-cross-resistant chemotherapy in the adjuvant setting. In a trial from the MD Anderson Cancer Center, 110 patients who had received neoadjuvant VACP (vincristine, doxorubicin, cyclophosphamide, and prednisone) without $\mathrm{pCR}$ were randomized to further treatment with VACP or VbMP (vinblastine, methotrexate, and prednisone). Results demonstrated that further treatment with adjuvant was tolerable, with the suggestion of improved outcomes in the VbMP arm over the VACP arm (OS $65 \%$ versus $47 \%, P=0.06$ ) [18]. In the small ICARO 1 study, presented in abstract form at the 2006 San Antonio Breast Cancer Symposium, 48 patients with clinical T4 disease were treated with neoadjuvant PEV (cisplatin, epirubicin, and vinorelbine), surgery, radiation, adjuvant CMF (cyclophosphamide, methotrexate, and 5fluorouracil) for six cycles, and endocrine therapy if indicated. Individuals who did not achieve pCR at the time of surgery $(n=35)$ were randomized to observation $(n=28)$ or reinduction therapy with epirubicin and sequential docetaxel $(n=7)$. At a median follow-up of 72 months, DFS and OS were significantly better in the reinduction group than in the observation group (100\% versus 53\%, $P=0.009 ; 100 \%$ versus 68\%, $P=0.05$, respectively) [19]. Although provocative, the numbers in this trial are too small for any definitive conclusions to be drawn. However, both studies suggest that further non-cross-reactive treatment may be of value. The GeparTrio study examined the effect of providing a non-cross-reactive regimen (NX; vinorelbine and capecitabine) to individuals without evidence of clinical response after two cycles of preoperative TAC (docetaxel, doxorubicin, cyclophosphamide). In the 74 patients studied, pCR rates were not significantly improved with the noncross-reactive NX regimen compared with those receiving further TAC (3.1\% versus 7.3\%) [20]. Despite the apparent lack of benefit from the non-cross-reactive regimen in this setting, the role of a novel regimen for non-responders completing an entire, rather than an abbreviated, course of preoperative treatment is unknown, as are long-term effects on outcome. 


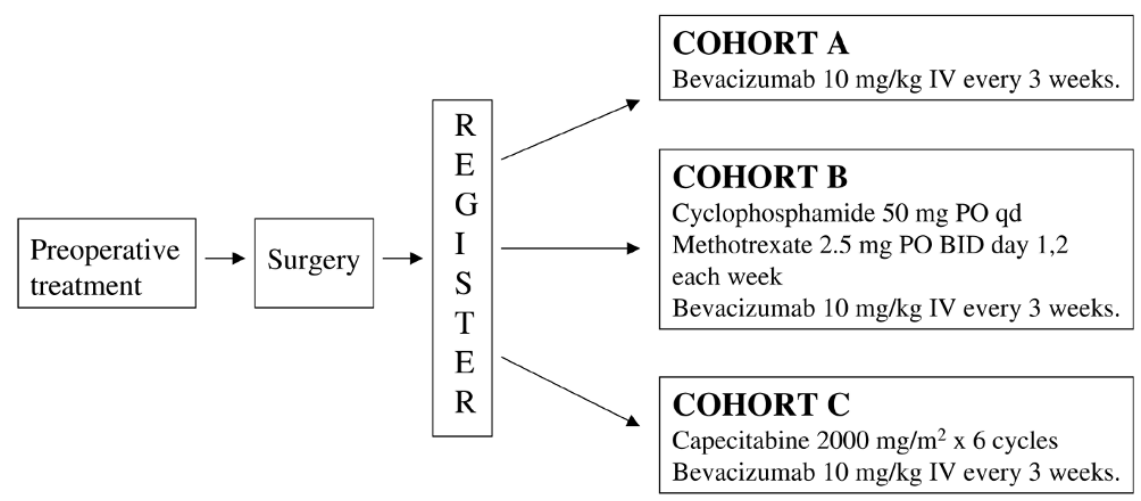

Schema for multicenter pilot study of angiogenesis inhibitor therapy after neoadjuvant chemotherapy. BID, twice a day; IV, intravenously; PO, orally; qd, once daily.

An alternative mechanism for targeting chemotherapyresistant disease may be through novel treatment approaches, such as the use of angiogenesis inhibitors. The primary agent in this class, bevacizumab, has already demonstrated marked benefits in several types of solid tumor [21,22]. In metastatic breast cancer, initial examination of bevacizumab with capecitabine chemotherapy in pretreated disease showed an improved response rate that did not translate into improved DFS or OS [23]. Subsequently, the addition of bevacizumab to paclitaxel chemotherapy in the first-line setting showed improvements in both response and DFS [24]. Another tool in targeting angiogenesis, metronomic chemotherapy (continuous low-dose oral treatment with cyclophosphamide and methotrexate) has also demonstrated activity in the metastatic setting in combination with bevacizumab [25]. It is possible that other novel biological therapies that show promise in breast cancer treatment may also have a role in the post-preoperative setting.

An ongoing multicenter pilot trial, led by investigators at the Dana-Farber Cancer Institute, is evaluating the feasibility and toxicity of 1 year of angiogenesis inhibitor therapy for individuals with less than a pCR after neoadjuvant chemotherapy (see Figure 1). Eligibility includes residual invasive disease in the breast or lymph node tissue after treatment with neoadjuvant, and receipt of a standard chemotherapy regimen, either preoperatively or postoperatively. In the first of three cohorts, 40 women have been treated with 1 year of adjuvant bevacizumab. This cohort included 45\% women with stage III disease at diagnosis and $58 \%$ who were hormone-receptor positive. Therapy was generally well tolerated, with treatment-related toxicities including mild arthralgias (50\%), headache (50\%), epistaxis (20\%), and hypertension (23\%). Significant toxicities included one case of reversible posterior leukencephalopathy and one patient with gastrointestinal bleeding. With a median follow-up of 8 months, four patients have recurred, underscoring the high- risk nature of this population. Extended follow-up from this study will evaluate possible long-term cardiovascular side effects from prolonged bevacizumab exposure. Accrual continues in the two other cohorts, which offer 1 year of bevacizumab paired with oral chemotherapy, either 6 months of metronomic chemotherapy or six cycles of capecitabine. Data from this pilot study will be used to design a phase III trial for this patient population randomizing to one of three arms: 1 year of bevacizumab, six cycles of capecitabine, or a combination of the two [26].

\section{Conclusion}

Neoadjuvant chemotherapy is an evolving strategy for treating locally advanced and early stage breast cancer. One potential advantage of treatment with neoadjuvant therapy is the opportunity to use pathologic response at the time of surgery to stratify patients by risk and subsequently select those for whom further therapeutic recommendations may be beneficial. As yet, the use of such dynamic tumor response information to guide clinical recommendations is in the investigative stages. However, it is hoped that the design of future clinical trials will allow an objective assessment of this management approach.

\section{Competing interests}

EM receives research funding from Genentech; LC is on the Advisory Boards and Speakers' Bureau of Genentech and receives research funding from Genentech; $\mathrm{HB}$ is on the Speakers' Bureau of Genentech and receives research funding from Genentech.

\section{References}

1. Sachelarie I, Grossbard ML, Chadha M, Feldman S, Ghesani M, Blum RH: Primary systemic therapy of breast cancer. Oncologist 2006, 11:574-589.

2. Fisher B, Bryant J, Wolmark N, Mamounas E, Brown A, Fisher ER, Wickerham DL, Begovic M, DeCilis A, Robidoux A, et al:: Effect of preoperative chemotherapy on the outcome of women with operable breast cancer. J Clin Oncol 1998, 16:2672-2685. 
3. Wolmark N, Wang J, Mamounas E, Bryant J, Fisher B: Preoperative chemotherapy in patients with operable breast cancer: nine-year results from National Surgical Adjuvant Breast and Bowel Project B-18. J Natl Cancer Inst Monogr 2001, 96-102.

4. van der Hage JA, van de Velde CJ, Julien JP, Tubiana-Hulin M, Vandervelden C, Duchateau L: Preoperative chemotherapy in primary operable breast cancer: results from the European Organization for Research and Treatment of Cancer trial 10902. J Clin Oncol 2001, 19:4224-4237.

5. Bear HD, Anderson S, Brown A, Smith R, Mamounas EP, Fisher $B$, Margolese R, Theoret $H$, Soran A, Wickerham DL, et al:: The effect on tumor response of adding sequential preoperative docetaxel to preoperative doxorubicin and cyclophosphamide: preliminary results from National Surgical Adjuvant Breast and Bowel Project Protocol B-27. J Clin Oncol 2003, 21:4165-4174.

6. Bear HD, Anderson S, Smith RE, Geyer CE, Jr, Mamounas EP Fisher B, Brown AM, Robidaux A, Margolese R, Kahlenberg MS, et al:: Sequential preoperative or postoperative docetaxel added to preoperative doxorubicin plus cyclophosphamide for operable breast cancer: National Surgical Adjuvant Breast and Bowel Project Protocol B-27. J Clin Oncol 2006, 24:20192027.

7. Mazouni C, Kau SW, Frye D, Andre F, Kuerer HM, Buchholz TA, Symmans WF, Anderson k, Hess KR, Gonzalez-Angulo AM, et al: Inclusion of taxanes, particularly weekly paclitaxel, in preoperative chemotherapy improves pathologic complete response rate in estrogen receptor-positive breast cancers. Ann Oncol 2007, 18:874-880.

8. Rouzier R, Pusztai L, Delone S, Gonzalez-Angulo AM, Andre F, Hess KR, Buzdar AU, Garbay JR, Spielmann M, Mathieu MC, et al.: Nomograms to predict pathologic complete response and metastasis-free survival after preoperative chemotherapy for breast cancer. J Clin Oncol 2005, 23:8331-8339.

9. Gianni L, Zambetti M, Clark K, Baker J, Cronin M, Wu J, Mariani G, Rodriguez J, Carcangiu M, Watson D, et al:: Gene expression profiles in paraffin-embedded core biopsy tissue predict response to chemotherapy in women with locally advanced breast cancer. J Clin Oncol 2005, 23:7265-7277.

10. Perou CM, Sorlie T, Eisen MB, van de Rijn M, Jeffrey SS, Rees CA, Pollack JR, Ross DT, Johnsen H, Akslen LA, et al.: Molecular portraits of human breast tumours. Nature 2000, 406:747-752.

11. Sorlie T, Perou CM, Tibshirani R, Aas T, Geistler S, Johnsen H, Hastie T, Eisen MB, van de Rijn M, Jeffrey SS, et al:: Gene expression patterns of breast carcinomas distinguish tumor subclasses with clinical implications. Proc Natl Acad Sci USA 2001, 98:10869-10874.

12. Sorlie T, Tibshirani R, Parker J, Hastie T, Marron JS, Nobel A, Deng S, Johnsen H, Peisch R, Geisler S, et al.: Repeated observation of breast tumor subtypes in independent gene expression data sets. Proc Natl Acad Sci USA 2003, 100:8418-8423.

13. Rouzier R, Perou CM, Symmans WF, Ibrahim N, Cristofanelli M, Anderson K, Hess KR, Stec J, Ayers M, Wagner P, et al.: Breast cancer molecular subtypes respond differently to preoperative chemotherapy. Clin Cancer Res 2005, 11:5678-5685.

14. Carey LA, Dees EC, Sawyer L, Gatti L, Moore DT, Collichio F, Ollila DW, Sartor Cl, Graham ML, Perou CM: The triple negative paradox: primary tumor chemosensitivity of breast cancer subtypes. Clinical Cancer Res 2007, 13:2329-2334.

15. Symmans W, Peintinger F, Hatzis C, Kuere H, Valero V, Hannessy $B$, Green M, Singletary E, Hortobagyi GN, Pusztai L: A new measurement of residual cancer burden to predict survival after neoadjuvant chemotherapy. Proc Am Soc Clin Oncol 2006, 24:A536.

16. Paik S: Importance of obtaining tissue for research - a case study in NSABP B27. Presented at National Cancer Institute meeting 'Preoperative Therapy in Invasive Breast Cancer: Reviewing the State of the Science and Exploring New Research Directions', Bethesda, MD, 26-27 March 2007.

17. Kaufmann M, Hortobagyi GN, Goldhirsch A, Scholl S, Makris A, Valagussa P, Blohmer JU, Eiermann W, Jackesz R, Jonaz W, et al:: Recommendations from an international expert panel on the use of neoadjuvant (primary) systemic treatment of operable breast cancer: an update. J Clin Onco/ 2006, 24:1940-1949.

18. Thomas E, Holmes FA, Smith TL, Buzdar AU, Fyre DK, Fraschini G, Singletary SE, Theriault RL, McNeese MD, Ames F, et al.: The use of alternate, non-cross-resistant adjuvant chemotherapy on the basis of pathologic response to a neoadjuvant doxorubicin-based regimen in women with operable breast cancer: long-term results from a prospective randomized trial. J Clin Oncol 2004, 22:2294-2302.

19. Ionta M, Atzori F, Scanu A, Scanni A, Cortesi E, Calluri A, Di Costanzo F, Maiorino L, Minerba L, Massidda B, et al.: Induction and reinduction chemotherapy following standard treatment in stage IIIB breast cancer patients achieving less than pCR (<pCR) after primary chemotherapy: results from a pilot Italian study (ICARO 1). Breast Cancer Res Treat 2006, 100:S156

20. von Minckwitz G, Blohmer JU, Raab G, Lohr A, Gerber B, Heinrich $G$, Eidtmann $H$, Kaufmann M, Hilfrich J, Jackisch $C$, et al.: In vivo chemosensitivity-adapted preoperative chemotherapy in patients with early-stage breast cancer: the GEPARTRIO pilot study. Ann Oncol 2005, 16:56-63.

21. Hurwitz H, Fefrenbacher L, Novotny W, Cartwright T, Hainsworth J, Heim W, Berlin J, Baron A, Griffing S, Holmgren E, et al:: Bevacizumab plus irinotecan, fluorouracil, and leucovorin for metastatic colorectal cancer. N Engl J Med 2004, 350:23352342.

22. Sandler A, Gray R, Perry MC, Brahmer J, Schiller JH, Dowlati A Lilenbaum R, Johnson DH: Randomized phase II/III trial of paclitaxel (P) plus carboplatin (C) with or without bevacizumab (NSC \#704865) in patients with advanced non-squamous non-small cell lung cancer (NSCLC): an Eastern Cooperative Oncology Group (ECOG) Trial - E4599. N Engl J Med 2006, 355:2542-2550.

23. Miller KD, Chap LI, Holmes FA, Cobleigh MA, Marcom PK, Fehrenbacher L, Dickler M, Overmoyer BA, Reimann JD, Sing AP et al:: Randomized phase III trial of capecitabine compared with bevacizumab plus capecitabine in patients with previously treated metastatic breast cancer. J Clin Oncol 2005, 23:792-799.

24. Miller K, Wang M, Gralow J, Dickler M, Cobleigh M, Perez E, Shenkier T, Davidson N: A randomized phase III trial of paclitaxel versus paclitaxel plus bevacizumab as first-line therapy for locally recurrent or metastatic breast cancer: a trial coordinated by the Eastern Cooperative Oncology Group (E2100). Breast Cancer Res Treat 2005, 94:S6.

25. Burstein H, Spigel D, Kindsvogel K, Parker L, Bunnell C, Partridge A, Come S, Ryan P, Gelman R, Winer E: Metronomic chemotherapy with and without bevacizumab for advanced breast cancer: a randomized phase II study. Breast Cancer Res Treat 2005, 94(Suppl 1):S6.

26. Mayer E, Miller K, Rugo H, Peppercorn J, Carey L, Ryabin N, Winer $\mathrm{E}$, Burstein $\mathrm{H}$ : A pilot study of adjuvant bevacizumab after neoadjuvant chemotherapy for high-risk breast cancer. Proc Am Soc Clin Oncol 2006, 25:56. 